\title{
Lemierre's syndrome with involvement of subclavian vein
}

\author{
Metin $\mathrm{M}^{1}$, Dogan $\mathrm{S}^{2}$, Yazici $\mathrm{H}^{3}$, Soy $\mathrm{FK}^{4}$, Dilci $\mathrm{A}^{5}$, Avcu $\mathrm{M}^{1}$ and Fidan $\mathrm{V}^{*}$ \\ ${ }^{1}$ Department of ENT, Ahi Evran Univ, Turkey \\ ${ }^{2}$ Department of ENT, Adyyaman Univ, Turkey \\ ${ }^{3}$ Department of ENT, Balıkesir Univ, Turkey \\ ${ }^{4}$ Department of ENT, Mardin Gov Hosp, Turkey \\ ${ }^{5}$ Department of ENT, Eskisehir Yunus Emre Gov Hosp, Turkey
}

\begin{abstract}
Lemierre's syndrome is a potentially fatal syndrome that is seen as a complication of orofarengeal infections and characterized by internal jugular vein thrombophlebitis, septic pulmoner embolism and other system embolism. Lemierre's syndrome is usually caused by Fusobacterium necrophorum, and mortality can be reduced with early diagnosis and appropriate antibiotic treatment. In this case, Lemierre's syndrome with subclavian vein involvement is shared and this case is different from previous reports.
\end{abstract}

\section{Introduction}

Septic thrombophlebitis of internal jugular vein is defined as Lemierre's syndrome [1,2]. This syndrome is caused by Fusobacterium necrophorum, gram negative anaerobic bacteria, found in oropharyngeal flora [3]. Lemierre Syndrome was first described by Andre Lemierre in 1936 [1]. Lemierre published a serie of 20 cases involving sepsis following pharyngeal infection. This complication, which was quite common before the use of antibiotics, was usually fatal and 18 of the 20 cases died $[1,4]$. After the discovery and widespread use of penicillin, this complication of diseases has become very rare and have been among the forgotten syndromes. However, in recent years, this syndrome has become more common due to decreased antibiotic use in tonsillopharyngeal infections. In the last 10 years, about 80 cases have been described in the literature and this number is quite high compared to past years. Possible reasons for this increase; less prescription of penicillins in the treatment of pharyngitis and tonsillitis and increased antibiotic resistance of fusobacterium [4-6]. In this case, an unusual case of Lemierre's syndrome in which ipsilateral subclavian vein thrombophlebitis accompanies with internal jugular vein thrombophlebitis following tooth extraction is presented.

\section{Case report}

Informed consent was obtained from patient before planning of this study. A 49-year-old male patient was examined at the ENT clinic because of swelling in the neck, fever at $39^{\circ} \mathrm{C}$, fatigue, respiratory distress, and chest pain. In the anamnesis of the patient; complaints of pain, redness and swelling started on the same side of the neck after tooth extraction 5 days ago. Although the dentist started oral ampicillin treatment 3 days ago, complaints were increased, trismus of the patient was started. Extensive swelling was detected in the submandibular region and parotide region on the left side in the physical examination. Fever and sensitivity were determined throughout the jugular veins (Figure 1). The patient had edema completely covering the left arm and pain on his left shoulder. In the laboratory tests; the number of white blood cells in the whole blood count: 13,300 uL, the number of platelets $350000 \mathrm{uL}, \mathrm{CRP}: 8,32 \mathrm{mg} / \mathrm{L}$, erythrocyte sedimentation rate: $120 \mathrm{~mm} / \mathrm{h}$. In the neck CT; there is a soft tissue thickening of the left masseter area, heterogeneous hyperdense area of $55 \times 28 \mathrm{~mm}$ in medial pterygoid muscle extending from premaxillary space to the anterior buccal area (Figure 2).Thrombus material reaching $1 \mathrm{~cm}$ thick in 3.5 $\mathrm{cm}$ segment at the level of juguler vein opening to subclavian vein on the left side was reported in the neck doppler ultrasound. It was

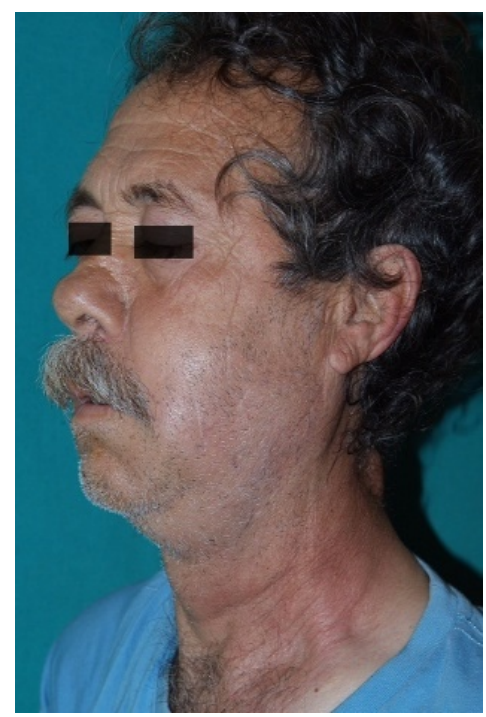

Figure 1. Clinical apperance of patient with Lemierre's Syndrome

Correspondence to: Associate Professor Vural Fidan MD, Department of ENT, Eskisehir Yunus Emre Gov Hosp, Turkey, E-mail: vuralf@mynet.com

Key words: Lemierre's syndrome, subclavian vein thrombophlebitis, Fusobacterium necrophorum

Received: December 20, 2017; Accepted: January 11, 2018; Published: January 15,2018 


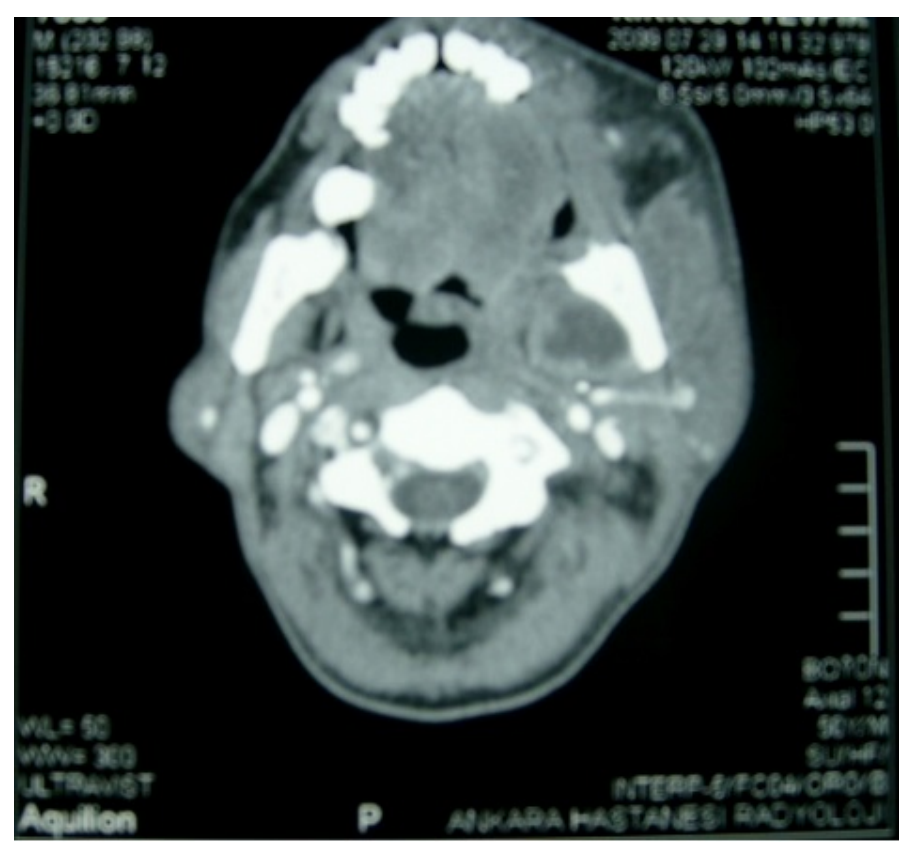

Figure 2. CT image of the neck

observed that the selected portions of the left subclavian venous were completely thrombosed. CT of the thorax showed pleural effusions in basal segments of both hemithorax and increased density in accordance with collapse-consolidation in adjacent basal segments. A number of parenchymal nodules measuring $12 \mathrm{~mm}$ in diameter were seen in the upper posterior and lateral of the lower lobe in the right lung.

The patient was empirically treated with ampicillin-sulbactam (IV), metronidazole (IV) at 4x500 $\mathrm{mg}$, subcutaneous enoxaparin sodium at $2 \times 40 \mathrm{mg}$ and oral acetylsalicylic acid at a dose of $1 \times 100 \mathrm{mg}$ in a dose of $4 \times 1.5$ gr. The purulent material and blood sample taken via punching were sent to the microbiology laboratory for bacterial culture and the result was reported as Fusobacterium necrophorum. The patient was referred to the Department of Cardiovascular Surgery to plan thrombectomy despite the clinical improvement at the end of four weeks of antibiotics and anticoagulant therapy.

\section{Discussion}

Fusobacterium necrophorum is found in the normal flora of oral cavity. Mucosal injury caused by another pathogenic microorganism facilitates the passage of fusobacterium to the parapharyngeal region [6]. Fusobacterium has some virulence factors like haemolysin, hemaglutinin, lipase, leukocidin, lipopolysaccharide [3]. Septic thrombophlebitis develops by local invasion of venules of tonsils or lymphatic channels [2]. The same process occurs in odontogenic infections, tooth extraction story is the main cause of mucosal injury in this case. When the patient was evaluated in our clinic, the absence of flow of abscesses or fluids in the mouth and in the gums suggested that the infection was spread by the vascular route. Bacterial spread is hematologically by septic embolism most often to the lungs, but joints, central nervous system, liver or kidney involvement are also described [3,7].

Clinical presentation of patients with Lemierre's syndrome can usually be with high fever, a one-week throat infection or another primary infection, fatigue, neck pain, tenderness and swelling throughout the sternocleidomastoid tract, dysphagia, and local abscesses. Septic embolism of the lungs occurs in $79-100 \%$ of the patients and associated cough, dyspnea, pleuritic chest pain and hemoptysis can be seen. Arthralgia occurs in 11-25\% of patients [7].

Jaundice is observed approximately $11-49 \%$ of patients and liver dysfunction is observed in the majority of patients. Coagulopathies may be occured, but not significant oftenly $[6,8]$. In this case, all symptoms except jaundice were present. In addition, the patient had trismus due to cellulitis and abscess formation in the parotid region. The most unique finding of this case was the presence of diffuse swelling completely covering the left arm of the patient due to left subclavian vein thrombophlebitis. There was not any case that describing Lemierre's syndrome with involvement of subclavian vein in our literature review.

The diagnosis of Lemierre's syndrome is based on clinical findings [9]. The first diagnosis that should be considered in a patient with sepsis and internal juguler venous thrombosis occurring one week after the oropharyngeal infection is Lemierre's syndrome. Leukocytosis, thrombocytopenia, high C-reactive protein are the main laboratory findings [3]. Multinodular infiltration of lung parenchyme and mild pleural effusion are seen on posterior-anterior chest X-ray typically $[7,9]$. Jugular vein thrombosis and cavitary lung lesions are observed in neck and thorax CT. Fusobacterium necrophorum can be isolated in blood and pus cultures [10].

Diseases such as infectious mononucleosis, pneumonia, endocarditis should be kept in mind in differential diagnosis [7]. Treatment should be directed against oral anaerobic and intravenous therapy should be given [4,9]. Fusobacterium necrophorum are $100 \%$ sensitive to metronidazole, ticarcillin-clavulanate, cefoxitin and imipenem. However, they are resistant to gentamicin and quinolones. In addition, resistance rates to penicillin and macrolides are $22 \%$ and $66 \%$, respectively $[9,11,12]$. The duration of treatment varies from 9 days to 84 days according to the treatment response of the patient $[9,13]$. Main treatment is intravenous antibiotic treatment for 2-3 weeks, clinical improvement is achieved and then the course is completed with oral antibiotic treatment for 5-6 weeks [7,9,11]. Surgical drainage is recommended if parapharyngeal, peritonsillary abscess, empyema, septic arthritis or other cavitary lesions are present in patients who can not be improved despite proper antibiotic treatment. Surgical drainage was performed twice for the abscess in the parapharyngeal region in this case.

Anticoagulant therapy in Lemierre's syndrome is controversial [14]. Different approaches have been reported in the literature. In the majority of recent pediatric cases, anticoagulant therapy was used. However, the number of cases is rather limited and there are no controlled studies [15]. Despite treatment with the appropriate antimicrobial agent for 48-72 hours, no clinical improvement is observed, and anticoagulant therapy should be initiated in cases such as concomitant thrombophilia and progression of jugular thrombosis [9,14]. In this case anticoagulant therapy is started immediately because of thrombosis of jugular vein and subclavian vein and pulmonary symptoms. Clinical improvement is the main criteria for determining the duration of anticoagulant therapy. Occasionally, in some cases persistent thrombosis may require surgical excision.

Early diagnosis and appropriate antimicrobial agent should be emphasized in the early period. With early antimicrobial agent treatment mortality is $0-18 \%$, whereas mortality after treatment on the fourth day is over $25 \%$. However, in cases of fusobacterium meningitis, the mortality rate is approximately $30 \%$. 


\section{Conclusion}

We report a rare case of Lemierre's syndrome with internal juguler venous involvement and subclavian venous involvement, which differs from the previously reported case of Lemierre's syndrome. This case is presented together with a literature review.

\section{References}

1. Lemierre A (1936) On certain septicemias due to anaerobic microorganisms. Lancet 1: 701-703.

2. Mesrar H, Mesrar J, Maillier B, Kraoua S, Chapoutot L, et al. (2017) Lemierre's syndrome: Diagnosis, exploration, treatment. Rev Med Interne. S0248-8663: 31177 33177 .

3. Rana MA, Kumar Y, Lashari AA, Mady AF (2017) Human Infection with Fusobacterium necrophorum without Jugular Venous Thrombosis: A Varied Presentation of Lemierre's Syndrome. Case Rep Infect Dis 5358095.

4. Chua SH, Ong SCL, Liew YH (2017) Variant of Lemierre's syndrome with internal jugular vein aneurysm. BMJ Case Rep 223371.

5. Bahall M, Giddings S, Bahall K (2017) Lemierre's syndrome: forgotten, but not absent. BMJ Case Rep 221203.

6. Riordan T, Wilson M (2004) Lemierre syndrome more than a historical curiosa. Postgrad Med J 80: 328-334.
7. Riordan $\mathrm{T}$ (2007) Human infection with fusobacterium necrophorum (necrobacillosis), with a focus on Lemierre syndrome. Clinic Microbiol Rev 20 : 622-659.

8. Armstrong AW, Spooner K, Sanders JW (2000) Lemierre's syndrome. Curr Infect Dis Rep 2: 168-173.

9. Miranda DL, Vasavada Z, Tanner C (2009) Lemierre syndrome following oropharyngeal infection: a case series. J Am Board Fam Med 22: 79-83.

10. Williams MD, Kerber CA, Tergin HF (2003) Unusual presentation of lemierre syndrome due to Fusobacterium nucleatum. J Clin Microbiol 41: 3445-3448.

11. Applebaum PC, Spangler SK, Jacobs MR (1990) B-lactamase production and susceptibilities to amoxicillin-clavulanate, ticarcillin, ticarcillin-clavulanate, cefoxitin, imipenem, and metranidazole of 320 non-Bacteroides fragilis bacteroides isolates and 129 fusobacteria from 28 U. S. centers. Antimicrob Agents Chemoter 34: 1546-1550.

12. Raggio BS, Grant MC, Rodriguez K, Cripe PJ (2017) Neonatal Lemierre Syndrome: Youngest Reported Case and Literature Review. Clin Pediatr (Phila) 9922817721156. doi: $10.1177 / 0009922817721156$.

13. Armstrong AW, Spooner K, Sanders JW (2000) Lemierre'ssyndrome. Curr Infect Dis Rep 2: 168-173.

14. Hoehn KS (2005) Lemierre syndrome: the contraversy of anticoagulation. Pediatrics 115: $1415-1416$

15. Schmid T, Miskin H, Schlesinger Y, Argaman Z, Kleid D, et al. (2005) Respiratory failure and hypercoagulability in a toddler with lemierre's syndrome. Pediatrics 116: e543-548.

Copyright: (C2018 Metin M. This is an open-access article distributed under the terms of the Creative Commons Attribution License, which permits unrestricted use, distribution, and reproduction in any medium, provided the original author and source are credited. 